Review

\title{
Biosensor Techniques Used for Determination of Telomerase Activity in Cancer Cells
}

\section{Eliona Kulla* and Evgeny Katz}

Department of Chemistry \& Biomolecular Science, Clarkson University, Potsdam, NY 13699-5810, USA.

* Author to whom correspondence should be addressed. E-mail: kullae@clarkson.edu

Received: 10 December 2007 / Accepted: 15 January 2008 / Published: 21 January 2008

\begin{abstract}
Measuring telomerase activity has proven successful for the determination of cancer in malignant somatic cells. Early conventional methods for the detection of telomerase activity include in vitro analysis via a primer extension assay, and the telomeric repeat amplification protocol (TRAP) assay. TRAP incorporates the polymerase chain reaction (PCR) step to increase the sensitivity of a given sample. However, research suggests that the TRAP technique suffers from false negative results, caused by failure of its PCR step. Other limitations of TRAP include the post-PCR steps involving polyacrylamide gel electrophoresis which are time inefficient. Thus, various efforts have been made to eliminate the PCR step of TRAP by using a variety of biosensor detection devices. This review mainly focuses on these alternatives including: optical, electrochemical, magnetic, and nanowire conductive signaling techniques to measure the telomerase activity produced via label free biosensor assay-via biocatalytic labels involving beacons, DNAzyme, ferrocenyl-naphthalene diimides, avidin-alkaline phosphatase and semiconductor quantum dots (QDs). These biosensor techniques are sensitive and provide precise and rapid results in the detection of telomerase activity.
\end{abstract}

Keywords: Biosensor, telomerase, electrochemical detection, biocatalytic labels, TRAP assay 


\section{Introduction}

Cancer is a group of diseases characterized by cell invasion, uncontrollable growth, and metastatic behavior into surrounding tissues or distant organs [1]. It has become the second leading cause of death in the United States, heart disease being number one [2]. Tremendous research in early-stage cancer detection [3] has increased in the last decade. Targets which have been studied include three different types of circulating biomarkers: DNA, RNA, and protein markers [4]. Today's technologies depict various highly-sensitive bioanalytical approaches for targeting these biomarkers [5]. The detection of telomerase, a ribonucleoprotein complex, has proven very affective as a cancer marker in tumors [6-7]. It has been found that about $80-90 \%$ of more than 950 primary tumors have been reported to express telomerase activity [8-11].

As normal cells divide, they reach the 'Hayflick limit' [12], meaning their finite life span, due to the end-replication problem [13] involving chromosome shortening. The role of the activated telomerase is to keep telomeres at the end of eukaryotic chromosomes, long enough to protect the termini of linear chromosomes from degradation and fusion [14]. Telomerase is a complex reverse transcriptase, consisting of GT-rich fragments which make up 130-210 kilobases of (TTAGGG) $)_{n}$ repeats. It is composed of two main subunits, a catalytic subunit defined as the human telomerase reverse transcriptase (hTERT) and a template of hTERT defined as the human telomerase RNA (hTR) [15]. Telomerase can be activated due to loss or a mutation of the tumor suppressor activity which can occur during cellular proliferation, immortalization, and transformation [15].

Research shows that human cells can undergo only a certain number of divisions in vitro until the DNA telomeric ends are successively shortened with every cell division [12]. In some cases senescence is overcome in the cell by carcinogen or virus-induced transformation, a complex process, which results in altered morphology and growth properties [16]. Most of the changed cells cease to divide and die, thus become immortal [17-19]. Telomerase activity was detected in unicellular eukaryotes, and it was considered to be responsible for the stability of telomere length [16-23]. Further early 1990s research on human cells, connected telomerase activity and held it responsible for the proliferation of human cancer cells [24]. Thus increased telomerase activity can provide important qualitative and quantitative results for cancer studies [24]. Its detection has been determined using various bioanalytical assays and techniques.

The very first detections of telomerase were direct telomerase activity assays, which included an oligonucleotide (as a substrate for the elongation process of telomerase), a cell extract, and a deoxynucleotide triphosphate (dNTP) mixture [25]. Using end-labeled substrate oligonucleotides or by incorporation of radioactive dNTP precursors, the activity of telomerase was analyzed [25-26]. This technique suffered from sensitivity problems. To measure the telomerase activity more affectively, a lot of the radioactive precursors had to be used [25-26]; this makes it unfavorable for routine use. An alternative method, which increased the sensitivity of telomerase for its detection, was proposed by Kim et al [8], and is known as the telomeric repeat amplification protocol (TRAP). The ribonucleoprotein enzyme telomerase uses its RNA as a template for the synthesis of TTAGGG repeats at the end of the chromosomes [20-22]. After each TTAGGG repeat unit, telomerase pauses to reposition on its internal RNA template before it synthesizes the next repeat [21]. 
The generated ladder consists of 6 nucleotide units at a given time. The added repeat units by telomerase can be analyzed by using PCR amplification which uses the telomerase substrate (TS) oligonucleotide as the forward primer, and an oligonucleotide which is able to anneal to the telomeric repeats (CX) as the reverse primer [8]. A recent review article published by Fajkus [27] summaries the TRAP technique and its limitations. A number of modified TRAP techniques were developed to account for its drawbacks [24-33]. Recent developments in bioelectronics and bionanotechnology [3750] result in novel analytical procedures allowing the analysis of telomerase activity with an extremely high sensitivity without the TRAP assay or the PCR step. The present review paper is aimed to give a short overview of these novel methods.

\section{Label free detection of telomerase activity during hybridization step}

Various modifications have been made to the classical TRAP method to account for the sensitivity limitations [27]. In most cases, high amounts of radioactive labels were added during the hybridization step, for the detection of telomerase using polyacrylamide electrophoresis; these proved cumbersome and in most cases inefficient [27]. Furthermore, the amplification via PCR in TRAP, fails by producing false-negative results [8]. Incorporations of label-free bioelectronic immunoassays [37] have been used to eliminate radioactive labels; however they still employ the PCR step. Alternatives, such as real-time biospecific interaction technologies [38] were rapidly considered, even shortly after the birth of TRAP [8].

More recently, using biosensor technology, Schmidt et al. devised an optical sensor with a biochemically modified surface to analyze the binding and catalytic properties of telomerase activity in real time [39] eliminating the TRAP assay. The method is label free, and it uses an integrated optical grating coupler where the amount of analyte is proportional to the change of the refractive index of the optical device [39].

First, a catalytic process was achieved by immobilizing the DNA oligonucleotides on the surface of the sensor. Phosphate groups were covalently-bound to the surface via the 5'-end oligonucleotide. Consecutively a complimentary DNA strand was added to the immobilized DNA. At the end of the complimentary DNA, on the 3'end, the DNA contained a short sequence which was free for the hybridization to occur. After the entire surface was converted with immobilized DNA and its complimentary stands, the overhanging 5'ends of the DNA were modified with phosphorothioates (PS). PS are known to provide a 10-fold higher affinity [40] for the binding of the telomerase to the primer binding site (Figure 1).

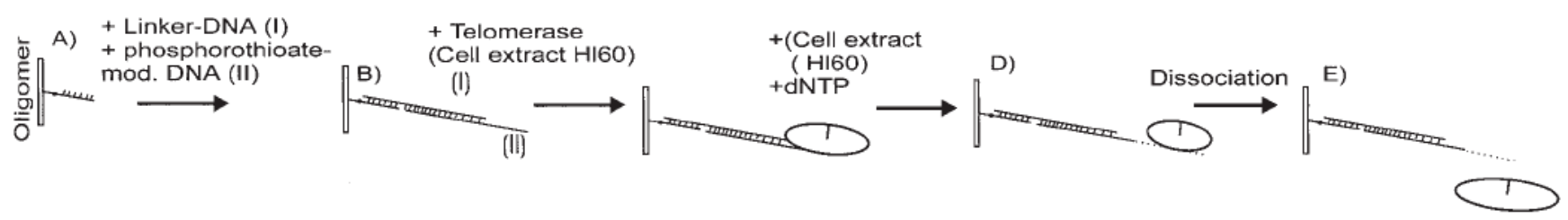

Figure 1. Scheme of the biochemical modification of the sensor surface [39, with permission]. 
The telomerase activity was measured upon the addition of cell extract containing telomerase, in a reaction buffer, in the presence of dNTPs mixture. The addition of the dNTPs allowed for the starting of the biocatalyzed synthesis process by telomerase [39]. The activity of the telomerase was measured by using a sensogram for determination of the surface loading in real time. The plain buffer forces the enzyme to dissociate from the DNA. The removal of the enzyme from the sensor surface is achieved by the addition of protenase K. Schmidt et al. determined the amount of nucleotides as incorporated by the telomerase enzyme. According to their experiments, 45 nucleotides were incorporated; this gave an enzymatic activity of 1 nucleotide/min [39]. Unfortunately the concentration of the telomerase was not determined since the immobilized enzyme could not be completely isolated from the sensor surface [39].

Additional experiments were conducted by Schmidt et al., demonstrating the importance of labelfree oligonucleotides during the hybridization step [41]. They studied the activity of label-free telomerase via total internal reflection fluorescence, on a fiber optical biosensor system [41]. Comparison was made when incorporating fluorescein isothiocyanate labels (FITC) which labeled the DNA probe before and after the elongation period of the telomerase sequence GGTTAG [41].

When the primer was elongated without the Oligo-FITC-labeled DNA-probe, the hybridization of the elongated repeat unit achieved a signal intensity of $0.43 \pm 0.005$ FI (Figure 2 (a)). The measurement of FI was obtained by taking the difference in FI signal from the baseline, before the hybridization takes place, and after the baseline, when the FITC labeled DNA-probe has been added. The difference in FI results due to the elongation portion achieved by the telomerase activity.

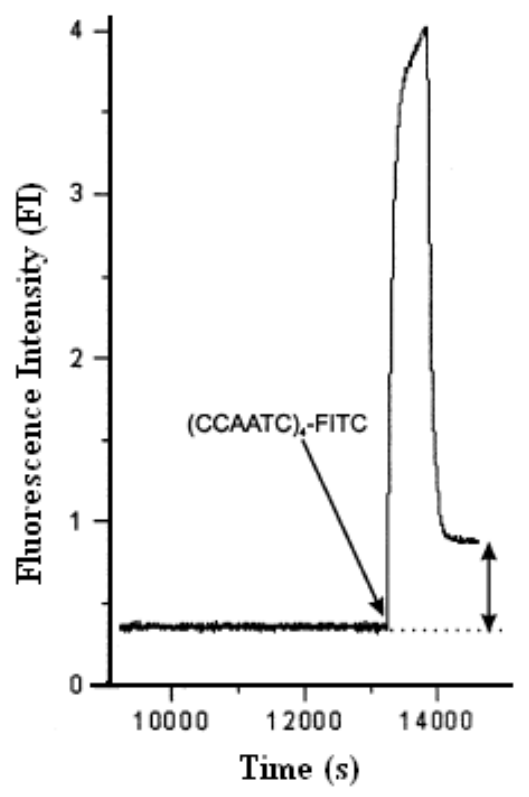

(a)

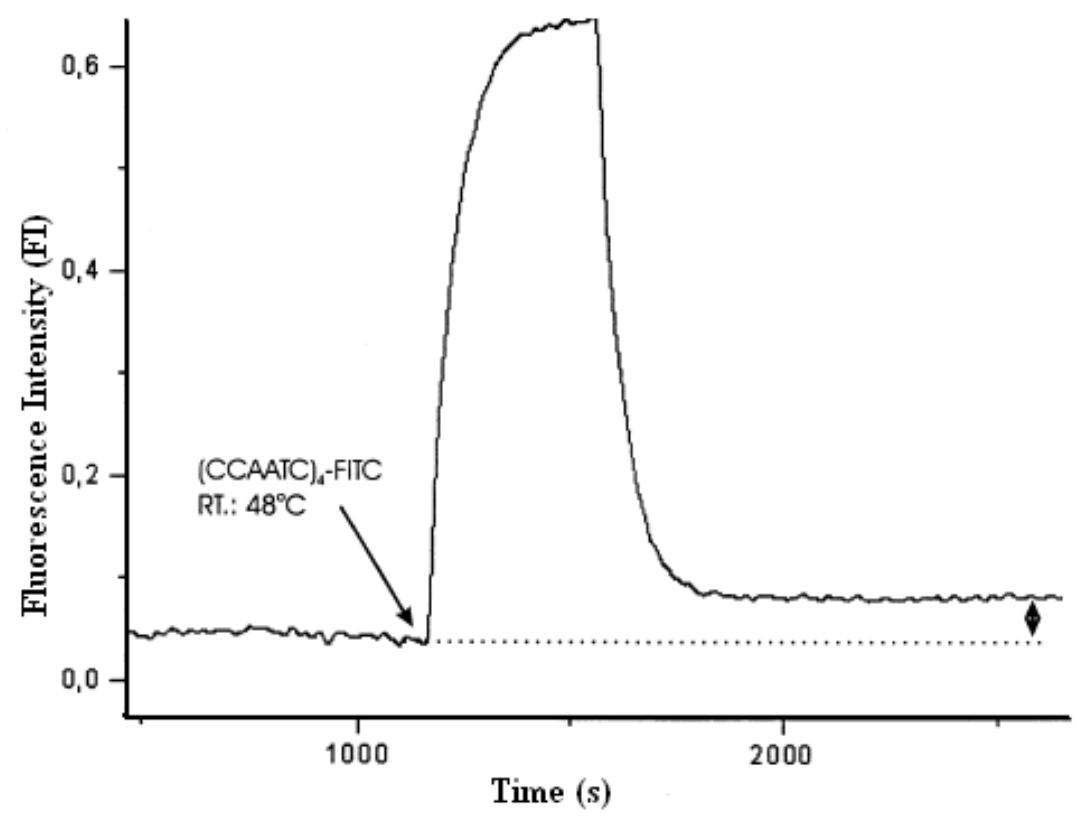

(b)

Figure 2. (a) Sensorgram of the telomerase activity using unlabeled dNTP and detecting the elongated primer by hybridization with FITC-labeled DNA-probe, and (b) Sensorgram of the integration of FITC-dUTP by telomerase on the surface of the fiberoptical sensor [41, with permission]. 
To determine the effect of the labeled nucleotides on the activity of telomerase, Schmidt et al mixed a UTP-FITC label with the dNTP before the elongation of the strand occurred. The elongated strand contained the fluorescent labeled species. Measurements of the fluorescence intensity were significant; the signal had dropped to $0.03 \pm 0.005$ FI (Figure 2(b)). This was about 12 times lower than the signal obtained earlier. This decrease in fluorescence intensity was attributed to the addition of the label which retarded the velocity of the telomerase [41]. As seen from these results, rationucleotides can inhibit the telomerase activity and slow down the rate of binding. This proves as a limitation to the usage of the TRAP assay.

Similar label-free methods as discussed above, have also been studied by Maesawa et al. utilizing the Surface Plasmon Resonance (SPR) biosensor detection technique [42]. This biosensor technique is similar to the grading coupler which was used by Schmidt et al. The analyte bound to the immobilized sample is proportional to the refractive index on the surface of the biosensor [42]. For his studies, Maesawa et al. constructed a telomeric repeat elongation (TRE) assay by immobilizing 5'-biotinylated oligomers (TS-5, B) oligomer on the streptavidin-pretreated dextran sensor chip surface. Extracts of telomerase were then allowed to bind to the oligomers for elongation of the immobilized oligomer to occur [42]. The binding of the telomerase did not require dNTP. The signals measured by the SPR are given in resonance units (RU) where $1 \mathrm{RU}=1 \mathrm{pg} / \mathrm{mm}^{2}$ [42]. Measurements were carried out in real time throughout the elongation and regeneration phases. Quantitative results were given in terms of elongation values (E-value) as obtained from a sensorgram. Rates of the telomerase activity were determined using these E-values [42].

Results for seven different types of cancer with varying forms were obtained [42]. These were compared to the elongation rate in mer/min of normal fibroblasts. As the concentration of telomerase activity was increased, so was the E-value (Figure 3(a)). Comparison of these cancer cells to normal fibroblast cells showed that the determination of telomerase was successful using this biosensor technique. As the concentration of the extracted telomerase extract in the cells was increased the evalue increased only for the cancerous cells (Figure 3(a)).

In another experiment using the TRE assay, a time dependence of $0.1 \mathrm{mg} / \mathrm{mL}$ of cell extract was measured. Results showed that the dependence was a linear concentration function of the e-value, for the specific cancer cells analyzed and also for the fibroblast cell lines (Figure 3(b)).

More specifically moles of DNA on the surface of the chip were measured by using a relationship where $1500 \mathrm{RU}$ are equivalent to $1.8 \mathrm{ng}$ of DNA (1.276 X $10^{-13} \mathrm{~mol}$ of TS-5'B oligomer). Therefore one base of all TS-5'B oligomers resulted in an increase of about 33.8 RU [42].

Additionally to the calculated rates of the elongation via telomerase, Maesawa et al. were able to correlate their elongation rates to those obtained by the TRAP assay. Results showed that the values were in good agreement with a correlation factor of 0.848 [42]. Thus, information via the rapid biosensor chip assays is just as useful as that obtained from the TRAP assay. 


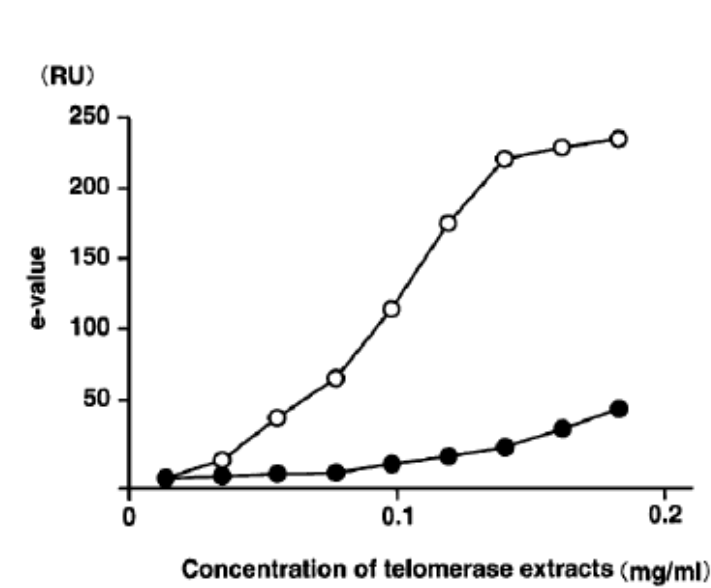

(a)

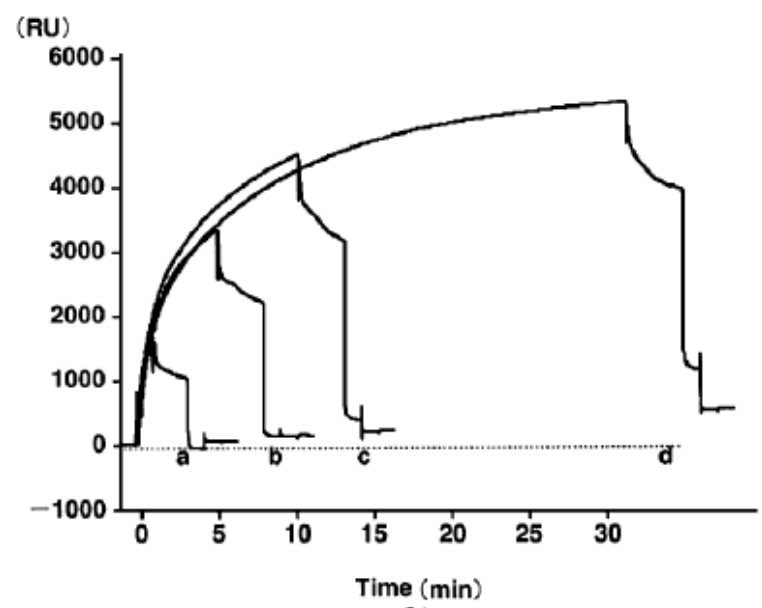

(b)

Figure 3. (a) Concentration as a function of e-value, ocolon cancer (SW480), normal fibroblast cell (SCS 2F0-C25) (b) Sensorgrams of SW480 at different infection times [42, with permission].

\section{Biocatalytic enzymes}

The main idea in most of the newly developed biocatalytic assays is generally the same. A nucleic acid which contains the RNA primer is chemically stabilized on a surface via sulfide bonds or is coiled up in the form of a catalytic beacon. Upon the presence of telomerase, the telomerization begins resulting in a long nucleic acid containing the repeat units [43-44]. The detection of the telomerized repeat units is achieved by complementary binding to a catalytic label, forming a catalytic complex , which is detected by electrochemical means. Different forms of biocatalytic labels and complexes formed thereof, are investigated.

\subsection{Catalytic beacons}

Xiao et al. [43] have used nucleic acid beacons with HeLa cancer cells, in the presence of the dNTP nucleotide mixture, where the telomerization started on the hairpin end of beacons [43]. Upon the opening of the beacon, a DNAzyme composed of a single-stranded guanine-rich nucleic acid and inserted hemin was activated revealing peroxidase-like activities [43]. This complex catalyzed the oxidation of 2,2'-azino-bis(3-ethylbenzthiazoline)-6-sulfonic acid (ABTS) in the presence of $\mathrm{H}_{2} \mathrm{O}_{2}$ producing color changes in the solution [43].

The beacon, as depicted in figure 4, contained two termini, designed for two functional nucleic acid components. One end of the hairpin structure contained a nucleic acid which included the base sequence, (Figure 4A), while on the other end, in the present of hemin, the base pair was hybridized (Figure 4B) in the hairpin configuration. The other end of the beacon contained a primer made up of a nucleic acid segment which was joined (Figure 4C). In the presence of HeLa cancer cells and a dNTP nucleotide mixture, the telomerization process started on the primer (Figure 4C) and the generated telomere (Figure 4(6)) hybridized to the complementary hairpin loop upon opening of the beacon [43]. 
Upon the addition of hemin to the opened hairpin structure, the ABTS oxidation by $\mathrm{H}_{2} \mathrm{O}_{2}$ determined the amount of telomerase activity. Using spectroscopic techniques, the chemiluminecent product was analyzed (Figure 5).

Results showed that the more colored product accumulated with time, the higher was the absorbance (Figure 5A). However, as can me seen from Figure 5A heat had a dramatic affect on the absorbance of of the HeLa cells. This decrease is said to be due to nonspecific binding of hemin to the reaction components [43]. The entire measurement can be performed in 8 minutes, which makes this procedure very time efficient [43].

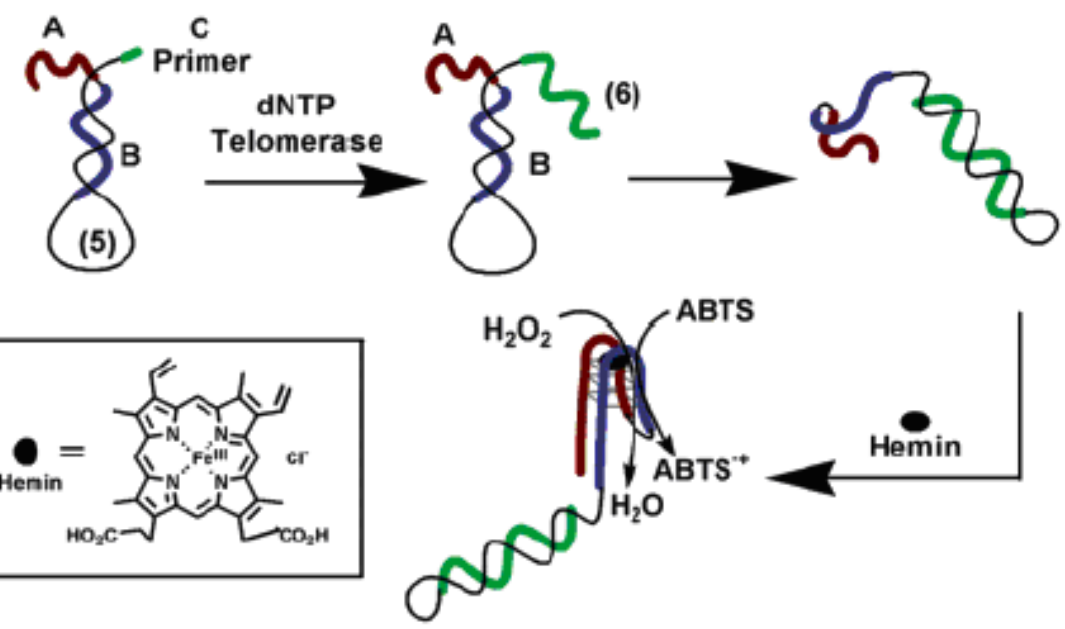

Figure 4. Analyzing telomerase activity via a functional DNA beacon, which selfgenerates a DNAzyme [43, with permission].
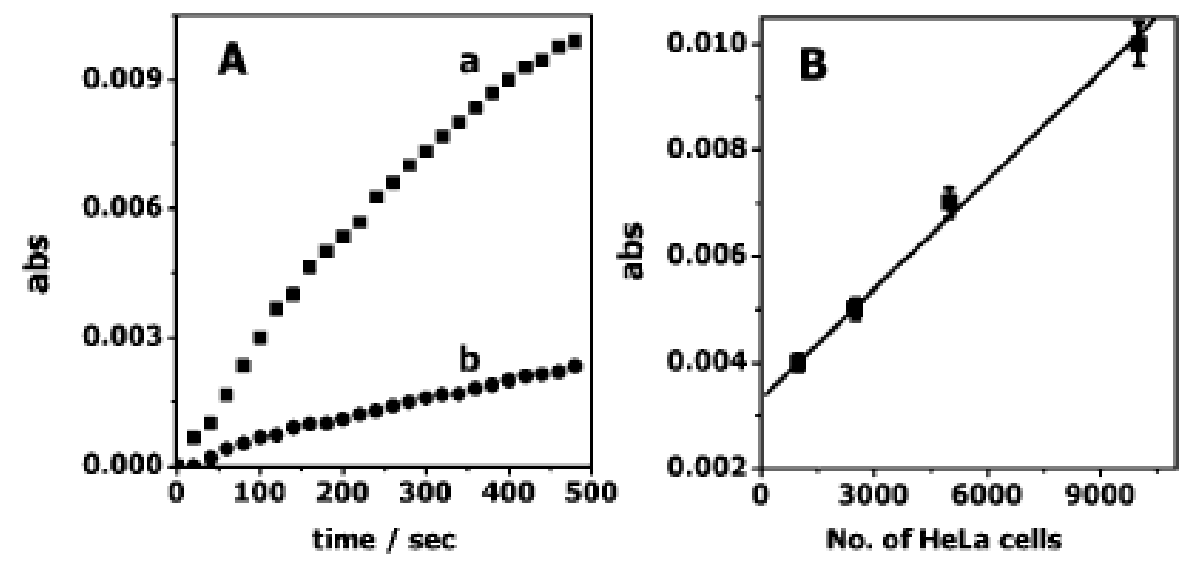

Figure 5. (A) Telomerase activity as analyzed by absorption measurements. a) 10000 HeLa cells and (b) 10000 heat-treated HeLa cells (95 $\left.{ }^{\circ} \mathrm{C}, 10 \mathrm{~min}\right)$. (B) Calibration curve of the absorbance of HeLa cells [43, with permission].

\subsection{DNAzyme}

Pavlov et al [44] used catalytic DNAzyme as a label for the amplified chemiluminescence detection of telomerase activity on a functionalized surface. In their work they use horseradish peroxidase (HRP) 
to mediate the process in the generation of chemiluminescence [44]. The representation of this method is depicted in Figure 6.

First, the telomere primer (5) was assembled on a gold surface and in the presence of dNTPs, the functionalized surface was interacted with the HeLa cancer cells. Telomerase was activated in the presence of HeLa cells, resulting in the amplification of (GGTTAG) $)_{n}$ units (6). Hybridizing the long sequence with a pre-designed nucleic acid (7) provided qualitative information about the amount of telomerase activity. The pre-designed supplement (Struct. I) was capable of forming a catalytic complex with hemin [44].

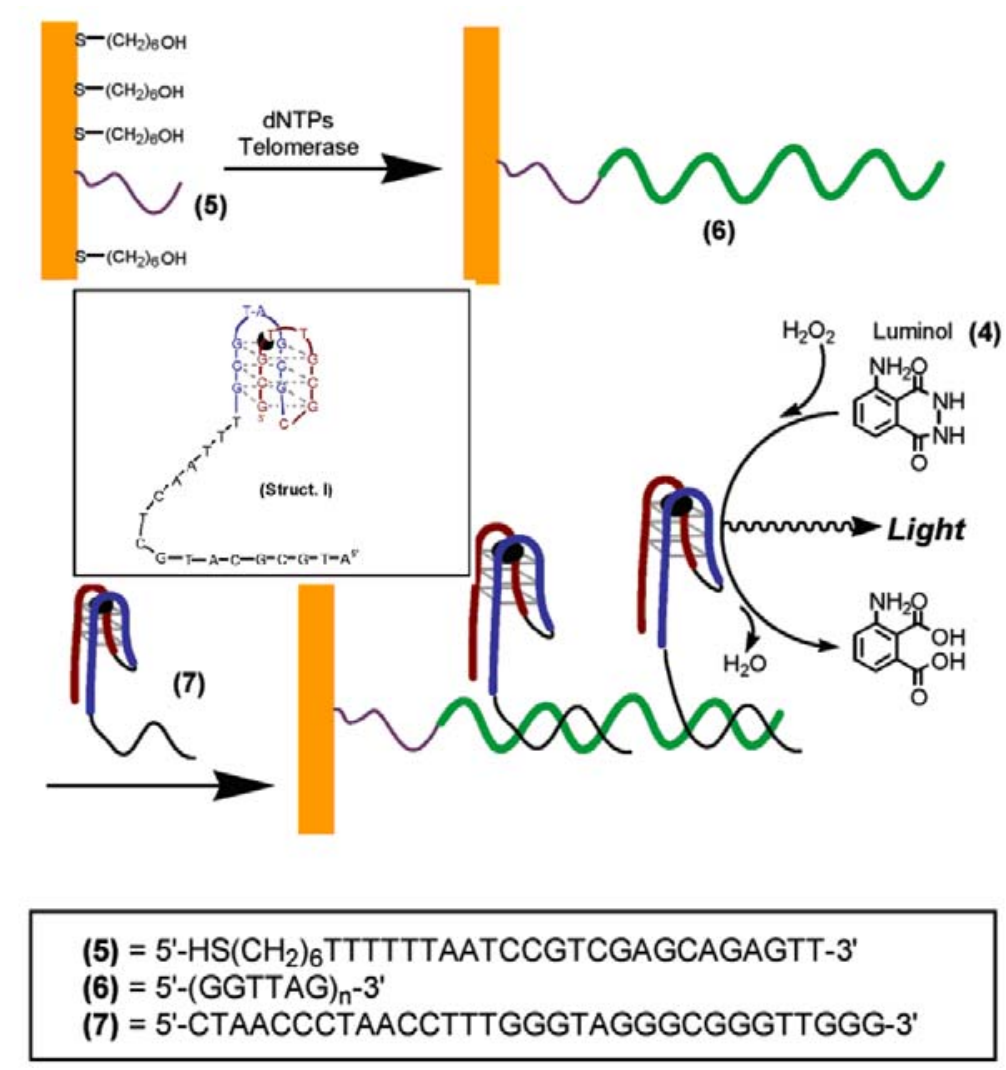

Figure 6. Analysis of telomerase activity using DNAzyme labels and chemiluminescence as a detection signal [44, with permission].

The chemiluminescence detection of telomerase activity was enabled by the biocatalytic oxidation of luminol by $\mathrm{H}_{2} \mathrm{O}_{2}$ and the light emission [44]. For the analysis of telomerase activity, first the hybridization was accomplished, followed by the catalytic DNAzyme which generated numerous photons (Figure 7).

Using this technique, results similar to the method using a catalytic beacon were observed [43]. When 10,000 HeLa cells were used, in standard conditions, the intensity of the emitted light, in this case the integrated photons, where more pronounced than then heat-treating the HeLa cells at $85^{\circ} \mathrm{C}$ for $10 \mathrm{~min}$ (Figure 7). As the temperature surrounding the HeLa cells was increased, the telomerase in the HeLa cells became thermally deactivated, resulting in the inhibition of the hybridization of (figure 7 (7)) and the inhibition of the biocatalyzed generation of the chemiluminescence. In addition, the heat- 
treated HeLa cells served as a good control for the employment of the DNAzyme as a label for the amplified detection of telomerase activity [44].

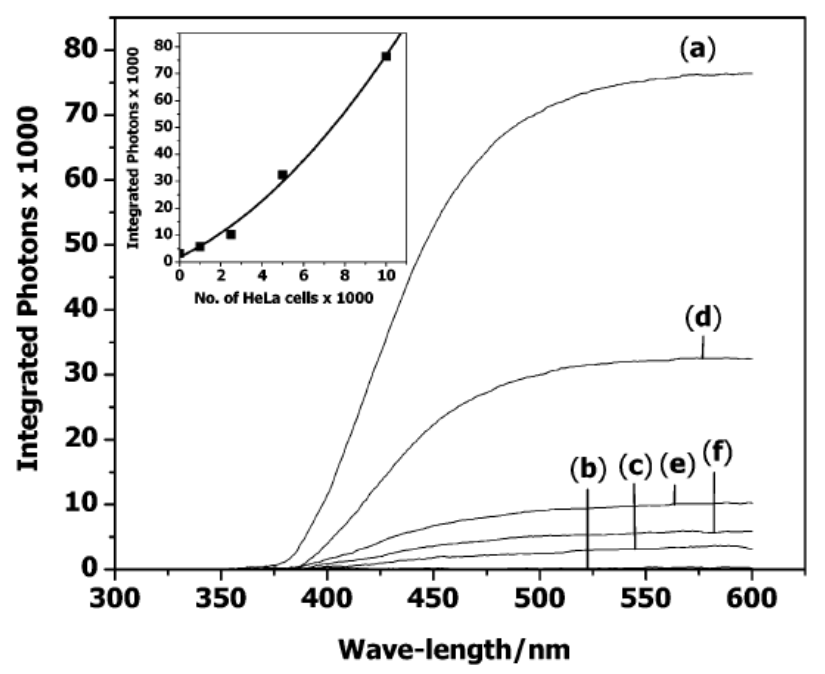

Figure 7. Integrated light intensities corresponding to the analysis of (a) $10000 \mathrm{HeLa}$ cells using DNAzyme (b) heat-treated HeLa cells (10 000) in the presence of the DNAzyme (c) 10, $000 \mathrm{HeLa}$ cells without the DNAzyme but after interaction with hemin 7 (d-f) 5000 , 2500, and $1000 \mathrm{HeLa}$ cells, respectively. Inset: Calibration curve corresponding to the analysis of different numbers of HeLa cells [44, with permission].

There are two advantages when using this detection setup. First, the nonspecific adsorption caused by telomerase on the nucleic acid interface or electrode surface is eliminated by the use of nucleic acids as biocatalysts [44]. Secondly, the use of the catalytic DNAzyme domain and the nucleic acid domain reduce the number of analysis steps which are involved using the TRAP essay [43-44].

\subsection{Ferrocenylnaphthalene diimide}

Sato et al [45], used a biocatalytic technique which provides an electrochemical signal which is based on ferrocenylnaphthalene diimide (figure 8A (1)) which can bind to tetraplex DNA by using telomerase from HeLa cancer cells (figure 8B). The principle of the method is the same as discussed above; however in this case, the telomerase activity is detected via a large amount of potassium ions studied via the quartz crystal microbalance electrochemical technique [45]. Under these conditions, the tetraplex DNA is formed on the electrode and ferrocenylnaphthalene diimide is concentrated on the tetraplex DNA [45].

Results show that in order for the detection of the telomerase to be made, telomere DNA has to assume a tetraplex structure. This is only possible under a large amount of potassium ions as detected by electrochemical techniques using spectroscopic means [45]. 
A

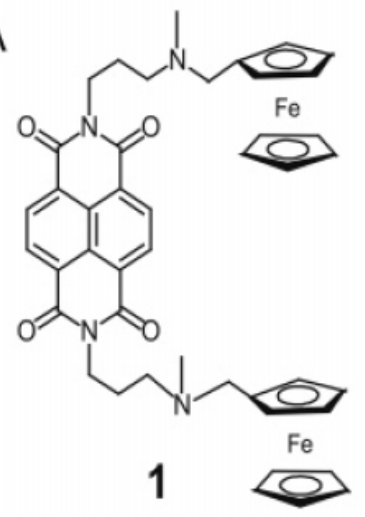

B

TS Primer

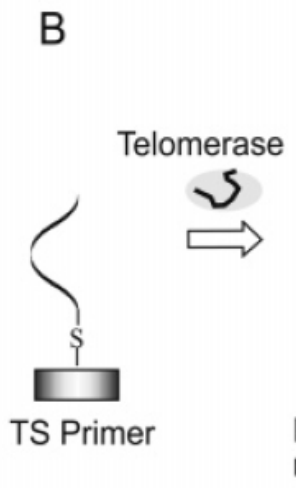

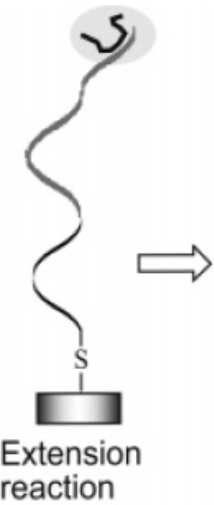

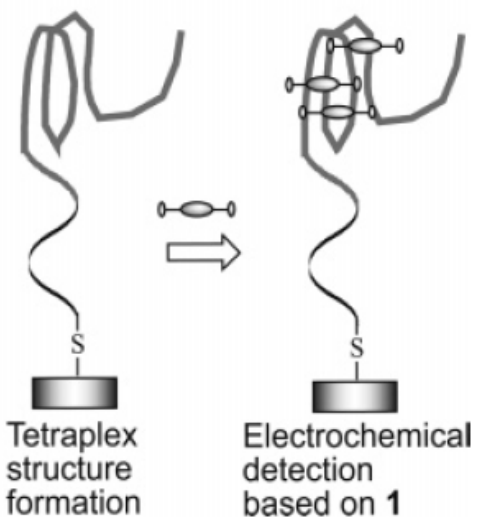

Figure 8. (A) Structure of ferrocenylnaphthalene diimine (1). (B) Principle methodology of the electrochemical assay. [45, with permission].

\subsection{Avidin-alkaline Phosphatase conjugate}

Pavlov et al demonstrated that the amplification pathway for the telomerase activity of HeLa cancer cells can be detected via the Avidin-alkaline Phosphatase label [46]. The set up (figure 9) consists of a thiolated nucleic acid (A1) bonded to a gold electrode via a sulfide bond. In the presence of dNTP and the HeLa cell containing telomerase were amplified on the primer bound to the surface. The telomerase sequence was reacted with a redesigned biotinylated nucleic acid (A2). Hybridization of the biotinylated nucleic acid to the complimentary telomere sequence occurred, followed by the association of the avidin-alkaline phosphatase (AP) conjuagate (A3) [46] to the biotin labels, the AP was catalyzed by oxidative hydrolysis of a 5-bromo-4-chrloro-3-indohyl phosphate (A4) to the insoluble derivative (5) which provided the amplification path [46]. Electrochemical techniques were used to monitor the amount of an insoluble product (A5) generated on the electrode surface (Figure 9A).

Using chronopotentiometry, an electrochemical technique which applies a constant current between the working electrode and the auxiliary electrode, provides the potential between the working electrode and the reference electrode changes, which are measured as a function of time [46]. Measurements were recorded in terms of over-potentials. The higher the over-potential, the greater the insulation of the electrode surface by the precipitate [46]. These results provide information about telomerase activity in HeLa cells, the greater the telomerization, the larger the resulting precipitate on the surface of the electrode and thus the higher the over-potential. When the experiment was performed with the pre-heated HeLa cells the over potential drastically decreased, suggesting that the telomerase was deactivated [46].

Another similar experiment was performed using a similar setup (Figure 9B), however in this experiment, the treatment of the functionalized electrode with the HeLa cells extract included not only dNTPs but also biotin-labeled dUTP. This biotin-labeled dUTP allows for the elimination of the hybridization step, since it was directly bound to one of the sites on the telomere repeat unit [46].

Using the chronopotentiometry technique, results with a slightly higher over-potential for the reduction of the redox labels was observed [46]. The increase in the over-potential suggested that using biotin-labeled dUTP results in a little more accumulation of the insoluble product on the surface 
electrode. Other methods for the analysis of telomerase activity were accomplished by microgravimetric quartz-crystal-microbalance $(\mathrm{BCM})$ measurements. Assemblies of nucleic acid monolayers on the Au-surfaces were characterized using this technique. Results show a similar trend; as the concentration of telomerase in the analyzed sample increases, the content of telomeres on the crystal surface also increases [46].

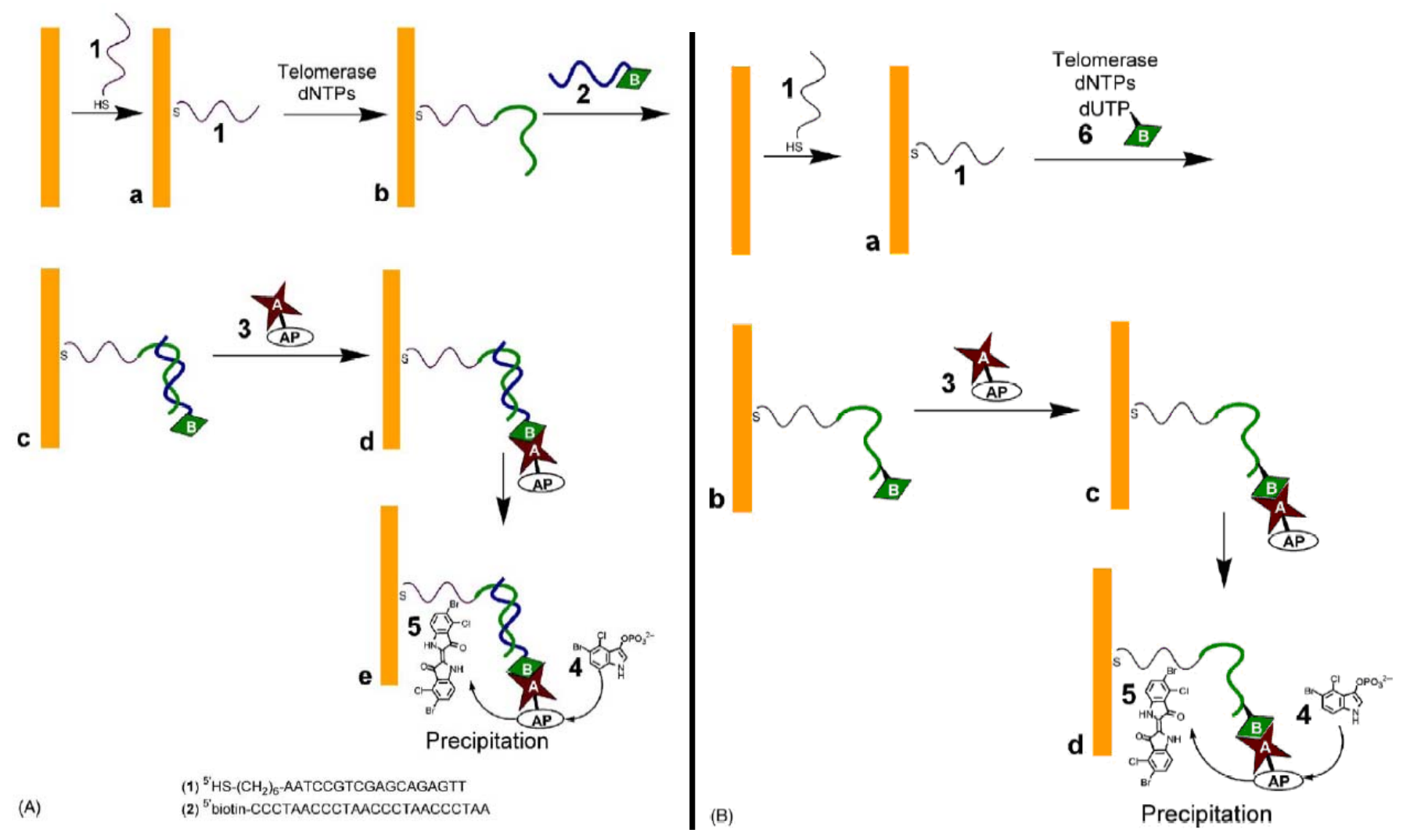

Figure 9. (A) The amplified analysis of telomerase activity by the hybridization of biotinlabeled telomere-repeat and the binding of avidin-alkaline phosphatase (5). (B) The amplified analysis of telomerase activity by the telomerase-induced generation of a biotinlabeled telomere, followed by the avidin-alkaline phosphatase conjugate (5) [46, with permission].

\section{Nanoparticles labels for detection of telomerase activity}

\subsection{Semiconductor quantum dots (ODs)}

Techniques mentioned above discussed, the detection of telomerase activity via the electrochemical means which included either a catalytic complex incorporated on a solid gold surface, or a complex using a catalytic beacon which opened and formed a DNAzyme complex when present under telomerase activity, that did not require a solid surface, or the formation of a precipitate [43-46]. Recent reports by Patolsky et al [47] use nanoparticles, semiconductor quantum dots (QDs) made from $\mathrm{CdSe}-\mathrm{ZnS}$ core-shell quantum dots, as solid surfaces to which the nucleic acid containing the RNA primer is mobilized (figure 10). These quantum dots exhibit photochemical centers for lighting-up the 
dynamics of telomerization which occurs on the nanoparticles by fluorescence resonance energy transfer (FRET) [48].

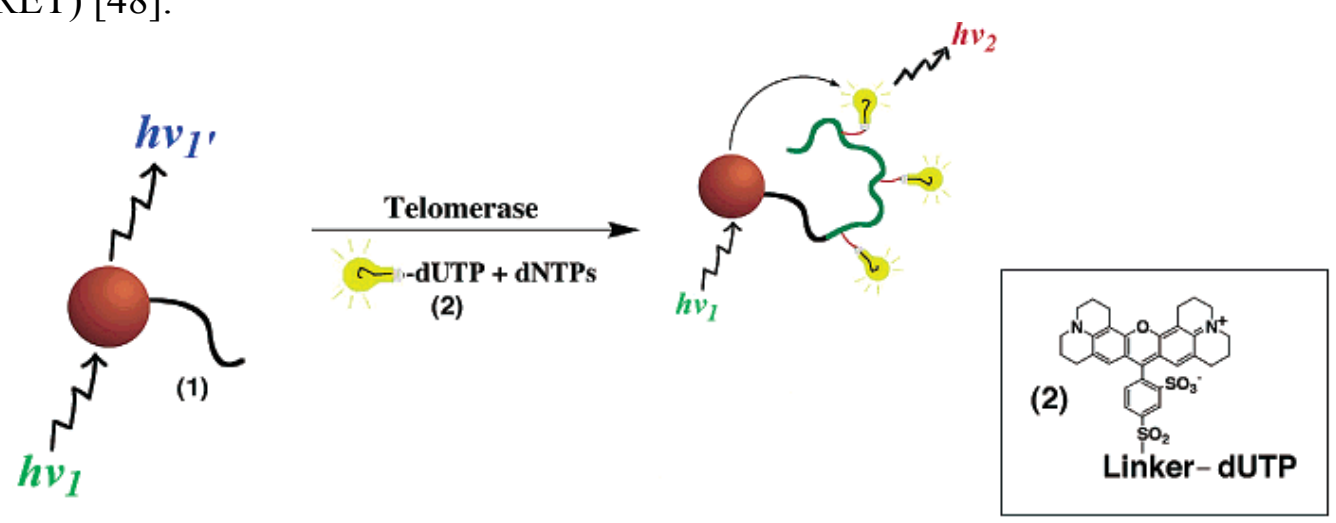

Figure 10. Telomerization on Nucleic Acid Funcionalized CdSe-ZnS QDs with the incorporation of Texas Red-labeled dUTP [47, with permission].

The attachment of the nucleic acid unit, containing the G-rich sequence recognized by telomerase for the generation of telomerase activity, contained a thiolated end group which made it possible for the attachment to the CdSe-ZnS QDs (figure 10(1)). In the presence of telomerase from HeLa cancer cells and a mixture containing dNTP and Texas-Red 14-dUTP (TR-dUTP, upon excitation of the CdSe-ZnS QDs, an emission peak at a wavelength equal to $560 \mathrm{~nm}$ was observed [47]. Emission of light decreases with time, since the light emitted is passed on and absorbed by the dye-modified dUTP, in the presence of the telomerase activity, showing an absorbance at a wavelength equal to $610 \mathrm{~nm}$. Therefore, the emission spectra at $610 \mathrm{~nm}$ increased with time (figure 11A).
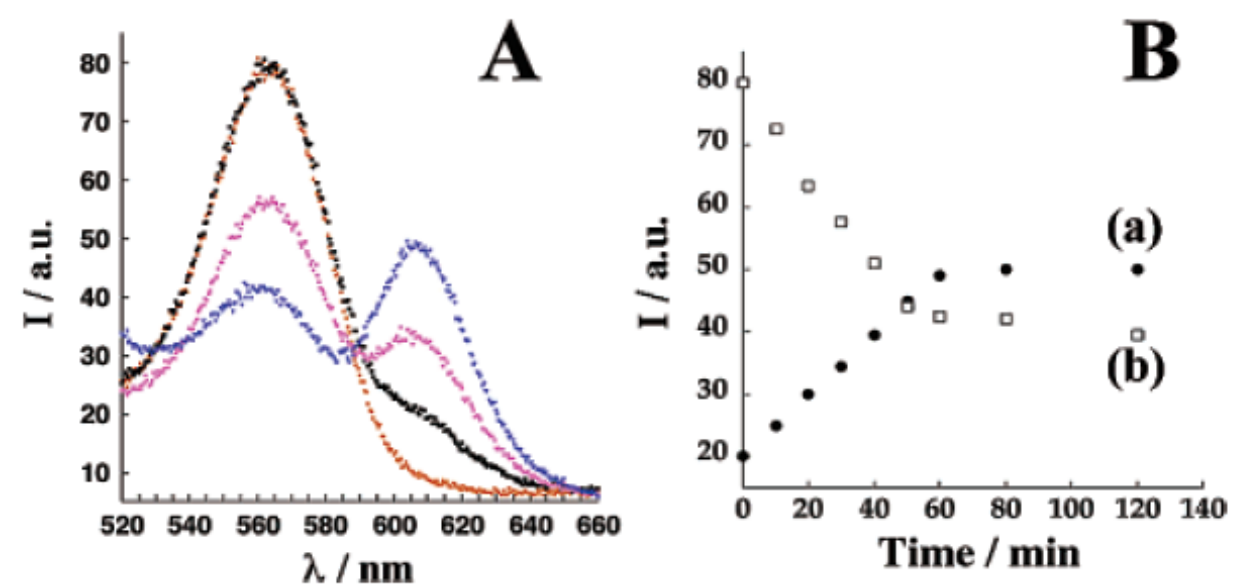

Figure 11. (A) Emission spectra upon the time-dependent DNA replication on the CdSeZnS QDs: orange, before addition of TR-dUTP; black purple and blue, after 1, 30, and 60 min of replication. (B) time-dependent decrease of the CdSe-ZnS emission (a) and the dye fluorescence increase (b), upon replication on the QDs [47, with permission].

Figure 11B shows a plot of the values of the emission spectra, intensity at the two wavelengths of interest as a function of time. A decrease of the QDs emission was observed as the intensity of the dye fluorescence increased (Figure 11B). Control tests were performed, empirically by changing one parameter and keeping the rest constant, to make these results conclusive. In addition, AFM images of 
the CdSe-ZnS QDs before and after telomerase treatment were recorded. Results provide qualitative information about the synthesized hinged DNA strands [47]. The picture corresponds to a telomeraseinduced elongation of about 1000 base units in the telomeric DNA chain (Figure 12B).

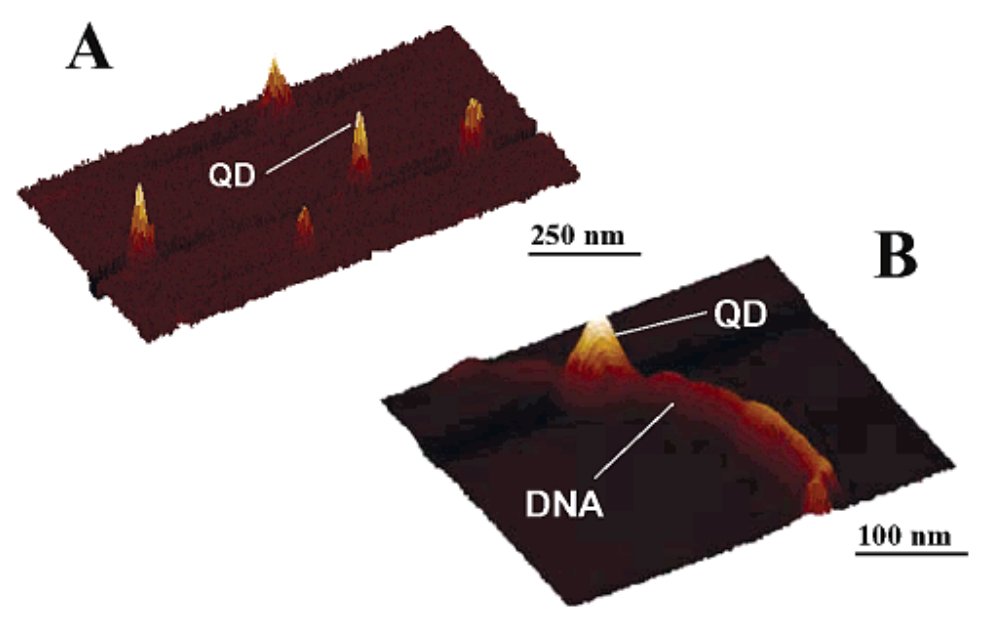

Figure 12. (A) AFM image of QDs before telomerase treatment. (B) AFM image of the CdSe-ZnS QD after 60min of telomerization [47, with permission]

\subsection{Magnetic Particles}

Weizmann et al [49] used a technique which incorporated magnetic nanoparticles in the presence of a magnetic field. This rotation of the magnetic particles by an external magnet allowed the particles to behave as rotating microelectrodes [49]. The biocatalytic processes at the surface of the electrode were controlled by convection [49]. This procedure enhanced biocatalytic transformations for detection of telomerase activity.

When attracting the magnetic particles, functionalized with telomerase-synthesized labeled telomeres close to the electrode, the rotation and electrogeneration of chemiluminescence resulted in exceptional sensitivity in the detection of telomerase activity [49].

The magnetic particles were functionalized with an amine and then activated with the bifunctional reagent 3-maleimidopropionic acid-N-hydroxysuccinimide ester. The mercaptohexyl-modified nucleic acid on one end contained a primer on the other end contains the G-rich RNA; the modified end was covalently linked to the magnetic particles [49].

The functionalized magnetic particles were treated with telomerase-containing cell extracts in the presence of a dNTP mixture that include biotin-labeled dUTP (Figure 11A). During telomerization, biotin labels were incorporated to the magnetic particles. The consequent binding of avidinhorseradish peroxidase (HRP) conjugate to biotin labels provided the biocatalytic conjugate into the telomere chains [49]. Using an external magnet, the magnetic particles containing the biocatalytic complex were collected at the bottom of the flask (excess materials washed away). After mixing the system with naphthoquinone-functionalized magnetic particles, the mixture of particles was introduced into an electrochemical cell that includes luminal [49]. When the quinine was reduced to hydroquinone, the electrocatalyzed reduction of $\mathrm{O}_{2}$ to produce $\mathrm{H}_{2} \mathrm{O}_{2}$ which was further used to mediate 
the HRP-catalyzed oxidation of luminol with the associated emission of light was observed [49]. The magnetic particles can covalently bond approximately 30,000 oligonucleotide units per particle [49].

Providing that the functionalized magnetic particles behaved as rotating microelectrodes, the speed of the rotation was proportional to the light emitted by the system (Figure 12(a)). The telomerase activity was directly related to the actual telomerase content in the sample.

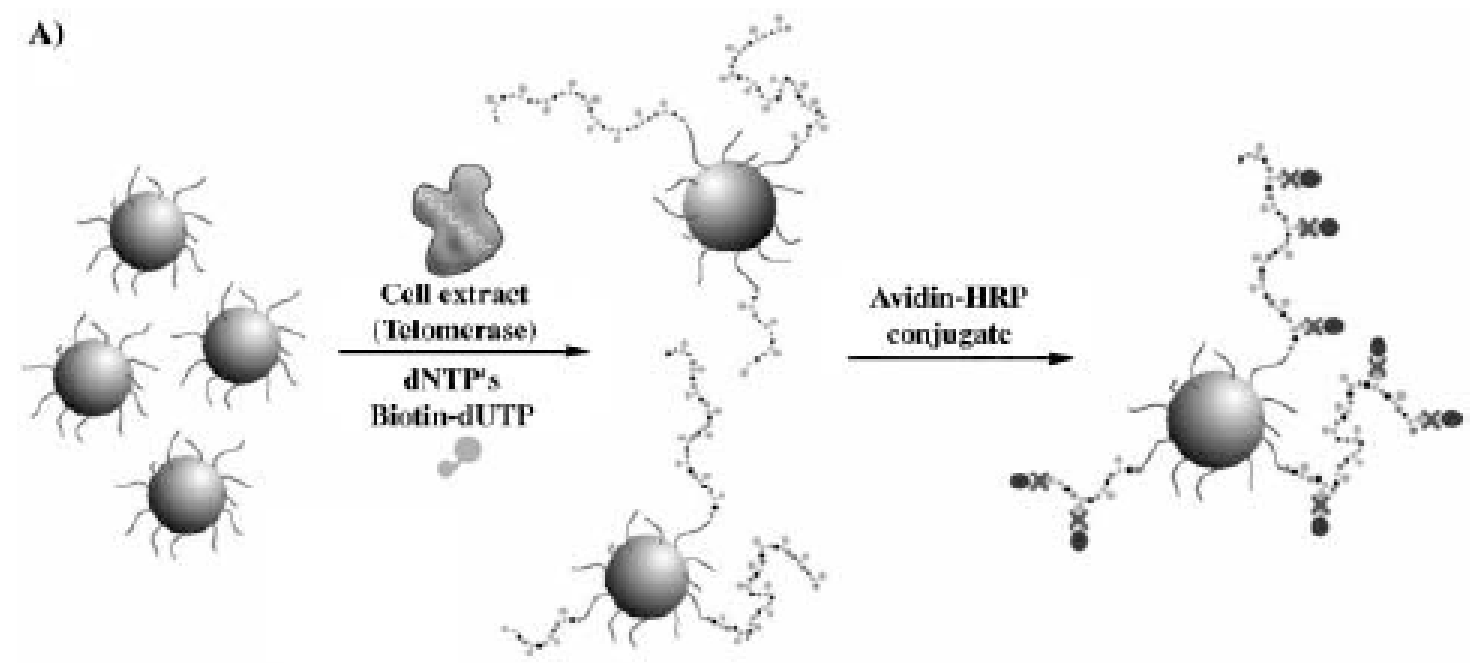

(1) $5^{\prime}$-HS-(CH $\left.{ }_{2}\right)_{6}$-TTTTTTAATCCGTCGAGCAGAGTT-3' Telomeric Repeats: TTAGGG
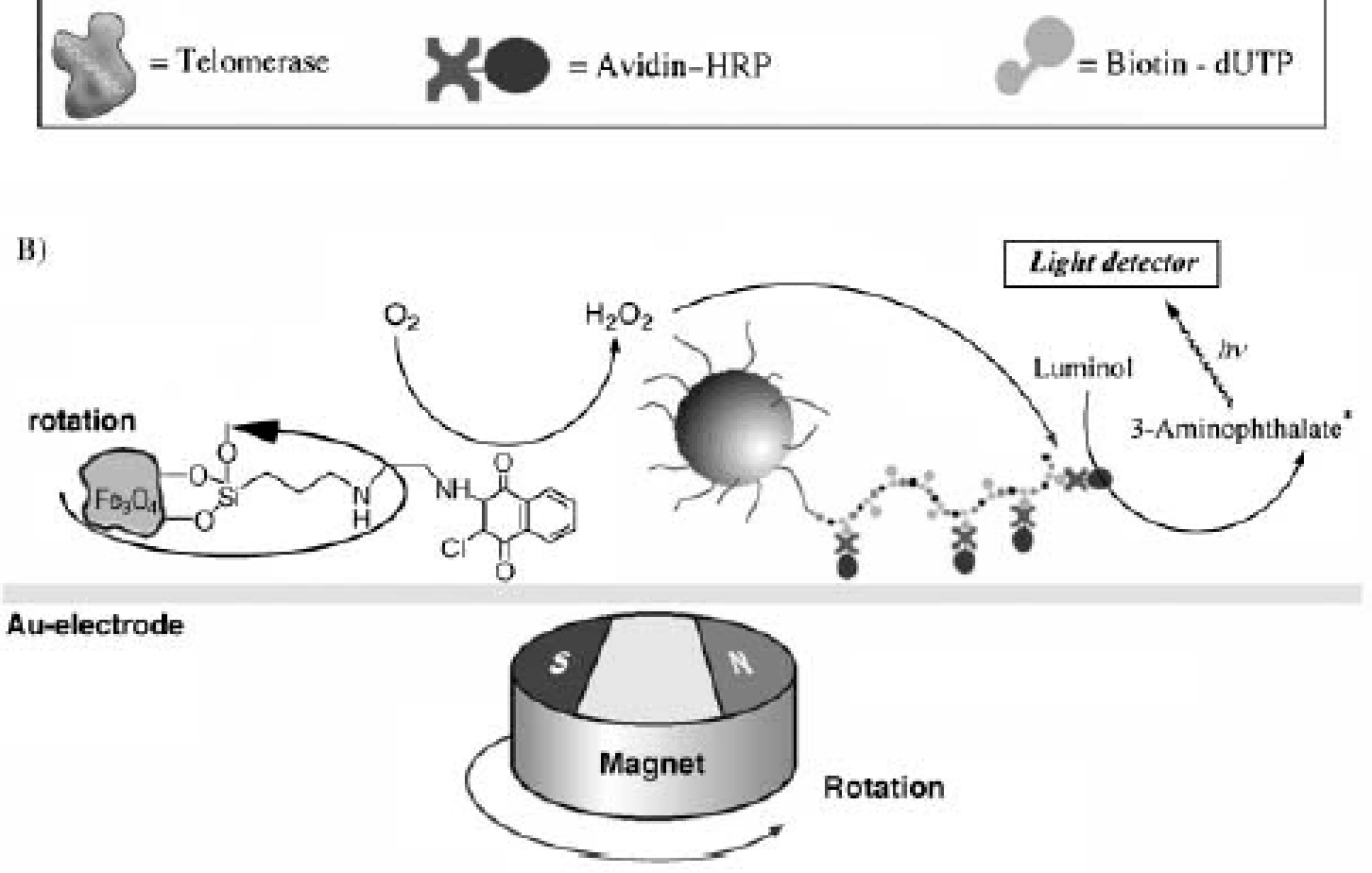

Figure 13. Amplified rapid detection of telomerase activity by multilabeled rotating magnetic particles. (A) Multilabeling of magnetic particles. (B) Generation of amplified chemiluminescence upon rotation of the magnetic particles[49, with permission]. 
A)

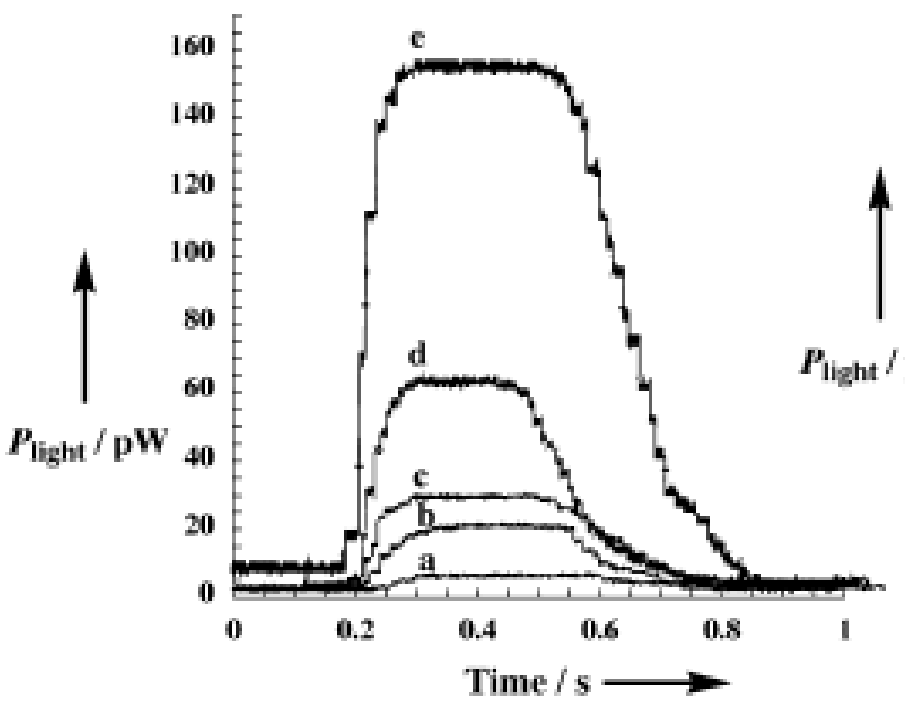

B)

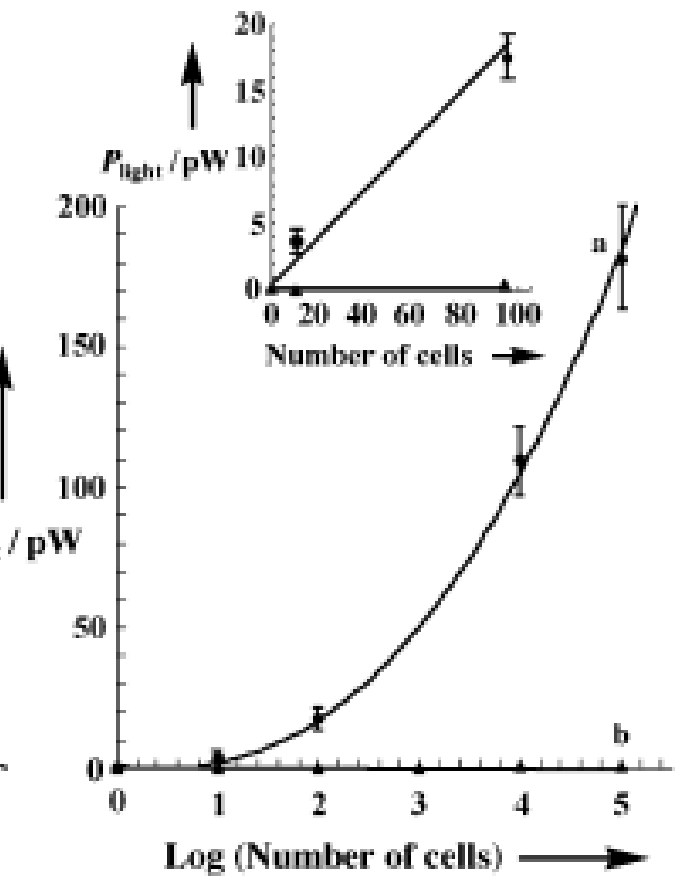

Figure 14: (A) Chemiluminescence intensities obtained with HeLa cell line extracts (100,000 cells), at different rotation speeds: a) $0 \mathrm{rpm}$, b) $20 \mathrm{rpm}$, c) $60 \mathrm{rpm}, \mathrm{d}) 400 \mathrm{rpm}$, e) $2000 \mathrm{rpm}$. (B) Calibration curve corresponding to chemiluminescence intensities of extracts containing: a) varying numbers of HeLa cells at a constant rotation speed of 2000 rpm; b) cell free control sample [49, with permission].

Experiments where the speed of the rotations of the magnetic particles was kept constant telomerase detetion in HeLa cells showed a linear relationship (Figure 14(b)). One limitation in this method could be a potential loss of the covalently linked complex from the magnetic particle thus resulting in some of the light not being recorded.

\subsection{Magnetic nanosensor}

An alternative approach from using rotating magnetic particles is to use magnetic particles for the construction of a magnetic nanosensor [50]. This approach was used by Grimm et al and it utilized the GGTTAG repeat sequence to construct thiolated oligonucleotides with the incorporation of magnetic nanoparticles (composed of cross-linked iron oxide with amide) for the detection of telomerase activity. The magnetic nanoparticles were confined in the sensor system with the thiolated oligonucleotides. These nanosensors were selected to identify 30-bp telomeric repeats when complimenting to the telomeric repeat unit [50].

Atomic force spectroscopy was used to examine the dispersion and the particle size of the magnetic particles in the nanosensors [50]. Results showed that the particles were monodispersed and their average size was $45 \pm 5 \mathrm{~nm}$, as measured by laser light scattering [50]. The incubation of the nanosensor with the telomeric repeats, as seen by atomic force microscopy, resulted in an assembly line of the nanosensors along the repeating telomeric sequence [50]. This was not observed for a controlled nonsense sequence, which suggests that the telomerase activity can be determined by the 
hybridization of an oligonucleotide, which contains nano-magnetic particles aligned in between the hybridized telomerase repeat sequences (figure 15).

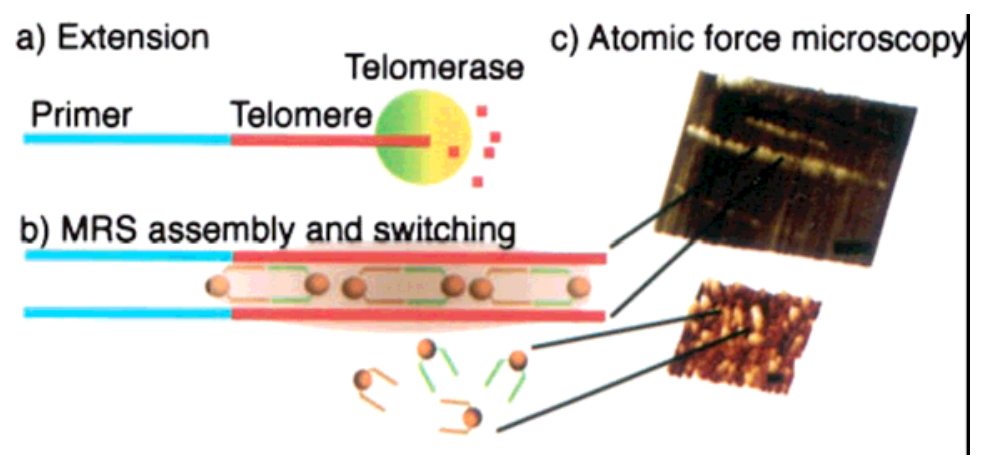

Figure 15. Telomerase magnetic nanosensor, assembly of nanoparticles is depicted by using atomic force microscopy [50, with permission].

Furthermore, relaxometry studies, where the effect of the hybridization on the magnetic spin-spin relaxation time (T2) was analyzed. Via strong and rapid magnetic switching ( $\delta \mathrm{T} 2)$, the relaxation of hybridized telomeric repeat units with the magnetic nanosensors was performed. Results from the magnetic switching were used to confirm the alignment of the nanoparticles in the hybridized telomeric repeats. In the control group where a nonsense sequence was used no hybridization occurred, this was detected by observing no measurable $\delta \mathrm{T} 2$ (Figure 16).

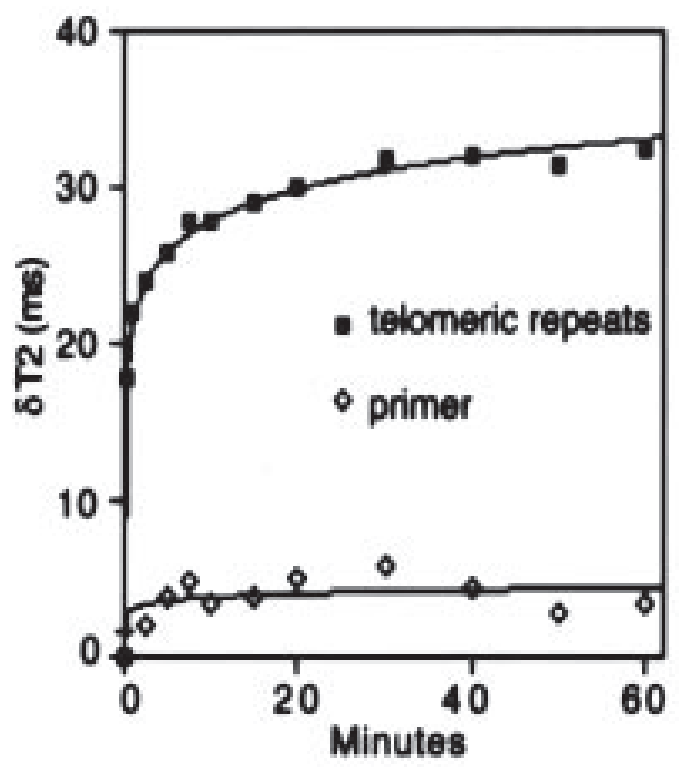

Figure 16. Induced magnetic $\mathrm{T} 2$ changes when adding telomeric repeat units or a primer [50, with permission].

The magnetic switching of 12 different cancer cells were monitored using magnetic sensors, where various controls and conditions were considered. Results were able to detect all 12 types of cancer by the magnetic properties of the sensors (Figure 17). Magnetic resonance imaging of plates were also collected showing the telomerase activity intensity (Figure 18). The range of the telomerase activity 
was identified in a unit scale ranging from $0-7.5(1$ unit $=2.94 \times \delta \mathrm{T} 2)$, where 7.5 was the highest detectable telomerase activity [50].

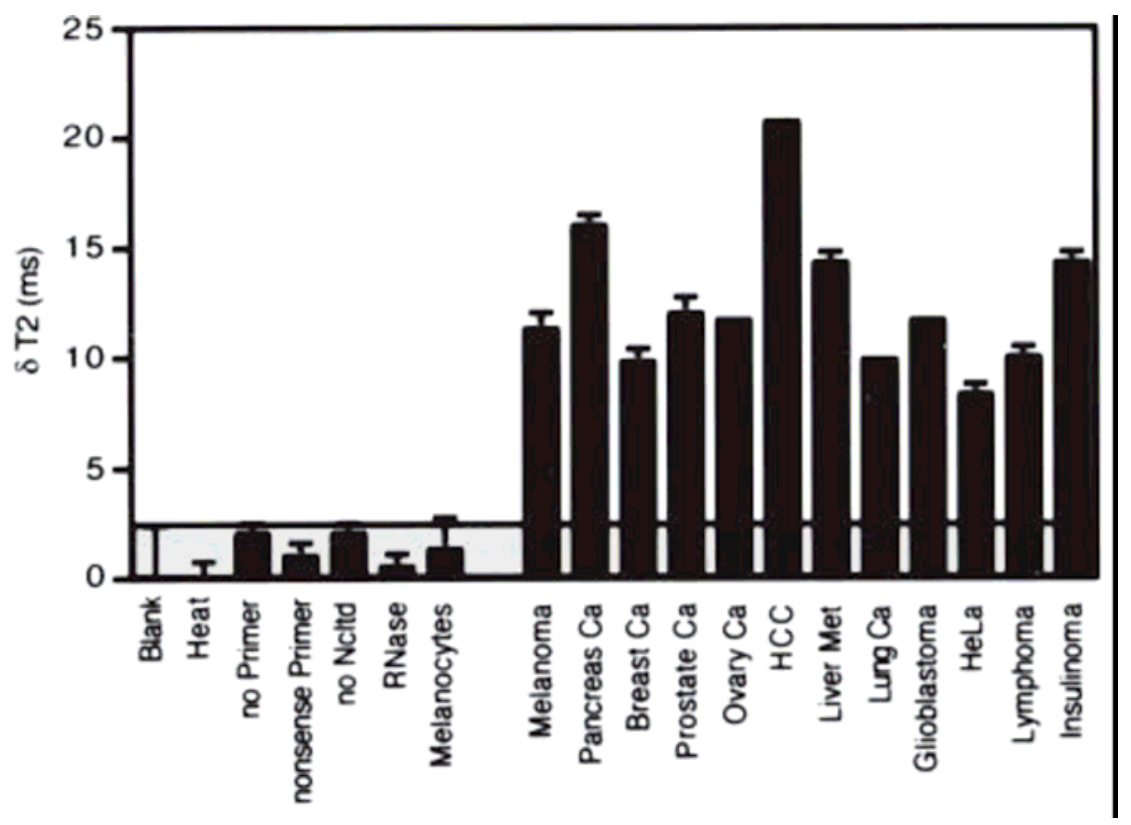

Figure 17. Detection of telomerase activity in different cell and tissue samples using the magnetic switch assay [50, with permission].

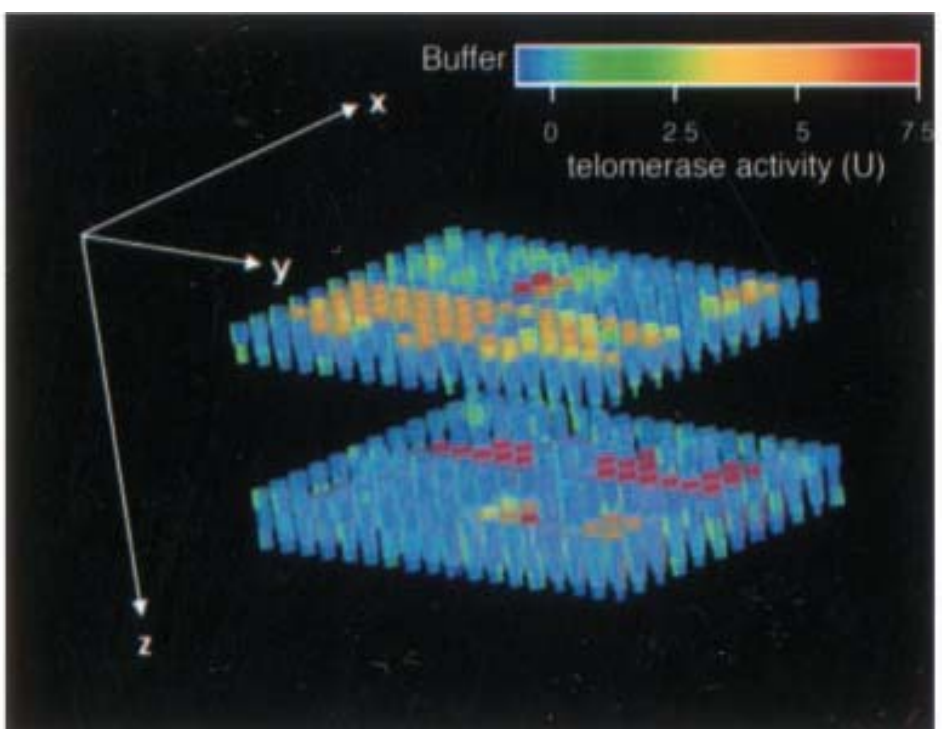

Figure 18. Detection of Telomerase activity using magnetic resonance imaging [50, with permission].

The sensitivity of this technique was also analyzed. Results based on a MR relaxometer-based assay were obtained [50]. They suggest that the minimum sensitivity of this nanosenser assay is about 100 attomoles when using samples which are recorded by relaxometers, however the sensitivity can go all the way down to the detection of a single molecule which is about 10 attomole by the magnetic force microscopy [50]. These results nicely compete with the sensitivity threshold of the TRAP technique with a correlation coefficient of 0.99 (Figure 19). 


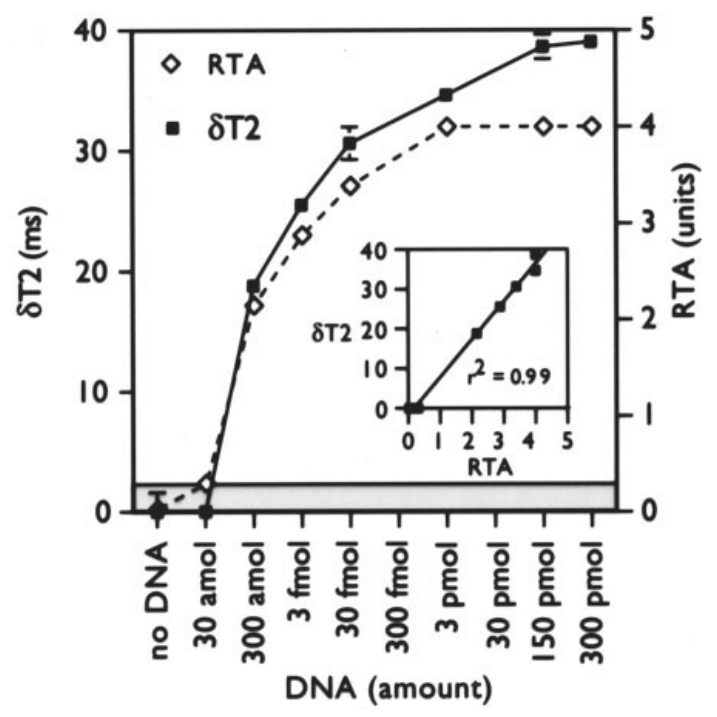

Figure 19. Comparison of the sensitivities of benchtop magnetic and standard TRAP assay using a 54-mer telomeric repeat [50, with permission].

In addition this technique is very fast when compared to TRAP technique [50]. As reported it takes about $150 \mathrm{~min}$ for the entire analysis to be performed [50]. Additionally it does not require a PCR step, labeled nucleotides, a post PCR gel electrophorises, or a solid phase. Some research [42] also suggests that not all telomerase activity is correlated to the telomerase length. Since this technique does not measure the telomeric length, it is a safer technique than TRE [42]. In addition, nanosensors are very useful techniques used for the screening of telomerase inhibitors and it can also be used to monitor telomerase regulation [50].

\subsection{Nanowire sensor chip}

Zheng et al. developed a label-free, highly sensitive nanowire sensor array which could be used for the detection of various cancer markers [51]. The sensor is a multiplexed electrical detector, which incorporates p-type antibody-functionalized silicon-nanowire and surface receptors into arrays [51].

The array is designed by attachment of monoclonal antibodies (mAbs) to the surface of the nanowire [51]. The silicon-nanowire surfaces are presented with aldehyde end groups on the nanowire surface. These aldehydic groups are then joined to mAbs, the free aldehyde groups are blocked by using ethanolamine [51].

This technique can be used with different types of silicon nanowires, such as p-type and n-types to form the electrical contacts by photolithography and metal deposition steps [51]. A conductance change was produced when a particular cancer marker protein was bound to the surface-linked mAbs if there was a matching receptor-modified silicon-nanowire device present [51]. However there will not be a conductance change produced if a silicon nanowire was lacking the specific antibody receptor [51].

This construction of this nanowire sensor was used for the determination of telomerase activity where the silicon-nanowire within an array which was first functionalized with oligonucleotide primers which would hybridize complimentary to the telomerase site [51]. The conduction of the 
nanowire can provide information about the presence or absence of telomerase in a particular cell extract [51]. The silicon micro array was selected to be made up of n-type nanowires which showed a decrease in conductance when successful binding of the telomerase to the oligonucleotide primers occurred [51]. The decrease in conductance was due to the telomerase being positively charged at a physiological $\mathrm{pH}$ [51].

These results are shown in the Figure 20 (a), where the conductance decreases with time after the addition of $100 \mathrm{HeLa}$ cells. In the contrary if the tissue extract is not cancerous, this meant that there was no telomerase and thus the conductance of the sample remained the same (figure 20 (b)).
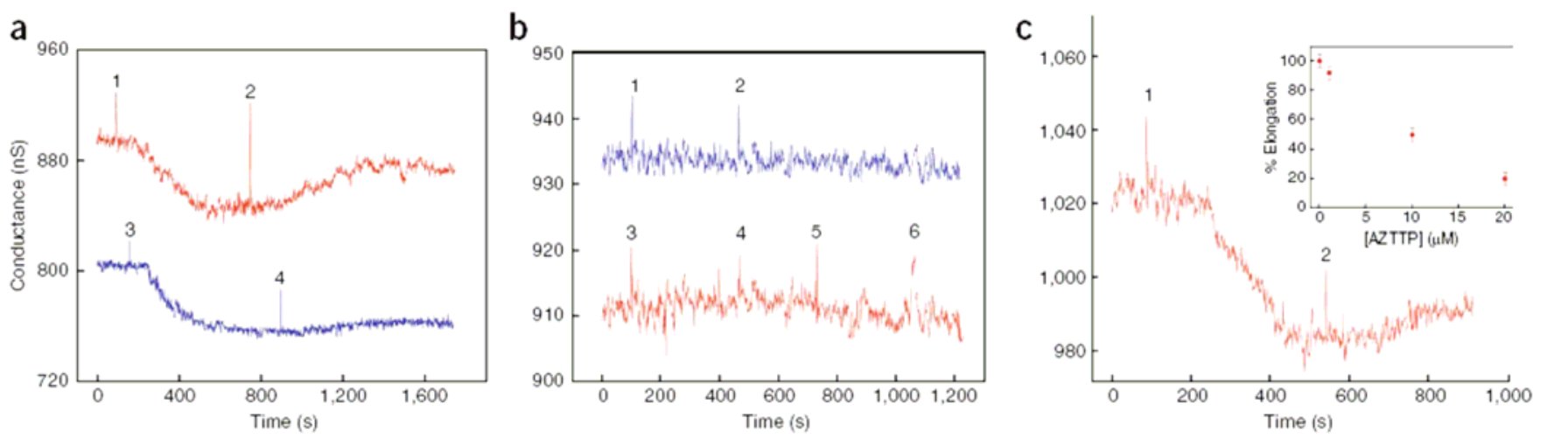

Figure 20. Detection of telomerase (a) Conductance-versus-time data recorded for oligonucleotide-modified p-type silicon-nanowire devices. (b) Conductance-versus-time data recorded for oligonucleotide-modified nanowire device. (c) Conductance-versus-time data recorded on a p-type silicon-nanowire device [51, with permission].

In addition, deoxynucleotide triphosphates (dNTPs) can be used for the determination of telomerase activity. The addition of dNTPs in the presence of telomerase results in the elongation of the telomeric unit, increasing conductance [51]. This increase in conductance is due to the incorporation of negatively charged nucleotides near the nanowire surface. This is also observed in Figure 20 (a), however as seen in Figure 20 (b) there is no increase observed after the addition of the dNTPs which means that there was initially no telomerase present.

The number of HeLa cells was found to be directly proportional to the conductance decrease in the Figure 20(a). The same was observed for the increase of the conductance after the introduction of the dNTPs. These results suggest that the conduction is also directly proportional to the telomerase concentrations [51].

In addition to studying the cancer in ideal cell extracts such as the HeLa cells, this technique was used for the detection of other protein cancer markers in clinical samples of blood serum where results obtained gave relevant information about the activity of the protein cancer [51]. In addition to all the other advantages which have been mentioned thoughtout the review, this particular nanowire sensor array is the first device which has been used for the detection of cancer in the human blood [51]. As seen from the results, the future promises a new technology with the availability of many nanomaterials. 


\section{Conclusions}

The TRAP method, even with its modifications, involves a lot of steps which make the procedure not as efficient. Their post PCR analysis using gels electrophoreses is also a tedious procedure. In addition, a few modifications of the TRAP assay eliminated the PCR amplification step which was responsible for the presence of Taq-polymerase inhibitors in clinical samples resulting in falsenegative results [27]. This review article provided other methods for the detection of telomerase activity which eliminate the PCR step and the TRAP assay combined. To date these methods include, label-free, various biocatalytic methods, and the incorporation of nanoparticles for the construction of biosensors. A review of these other methods suggests that these procedures are simple, efficient and accurate in the detection of telomerase activity. These techniques are versatile in detecting telomerase in various cancer cells.

\section{Acknowledgements}

Acknowledgement goes to the graduate course CM 725, Special Topics in Analytical Chemistry at Clarkson University, taught by Dr. Evgeny Katz, which initiated the preparation of this review paper.

\section{References and Notes}

1. Hiyama, E.; Hiyama, K. Telomerase as Tumor Marker. Cancer Letters. 2003, 194, 221-233; DOI 10.1016/S0304-3835(02)00709-7; PubMed 12757980.

2. Greenlee, R. T.; Hill-Harmon, M. B.; Murray, T.; Thun, M. Cancer Statistics. Cancer J. Clin. 2001, 51, 15-36.

3. Kosary, C. L. FIGO Stage, History, Histology, Histologic Grade, Age and Race as Prognostic Factors in Determining Survival for Cancer of the Female Gynecological System: an Analysis of Cancer of the Female Gynocological System: an Analysis of 1973-87 SEER Cases of Cancer of the Endometrium, Cervix, Ovary, Vulva, and Vagina. Semin. Surg. Oncol. 1994, 10, 31-46; DOI 10.1002/ssu.2980100107; PubMed 8115784.

4. Nalwa, H. S.; Webster, T. Cancer Nanotechnology: Nanomaterials for Cancer Diagnosis and Therapy; 2007American Scientific PublishersCalifornia.

5. Chaerkady, R.; Pandey, A. Quantitative Proteomics for Identification of Cancer Biomarkers. Proteomics Clinical Applications. 2007, 1, 1080-1089; DOI 10.1002/prca.200700284.

6. Zimmermann, S.; Martens, U. M. Telomers and Telomerase as Targets for Cancer Therapy. Cellular and Molecular Life Sciences. 2007, 64, 906-921; DOI 10.1007/s00018-007-6481-8.

7. Hiyama, E.; Gollahon, L.; Katacka, T.; Kuroi, K.; Yokoyama, T.; Gazdar, A. F.; Hiyama, K.; Piatyszek, M. A.; Shay, J. W. Telomerase Activity in Human Breast Tumors. J. Natl. Cancer. Inst. 1996, 88, 116-122; DOI 10.1093/jnci/88.2.116; PubMed 8537972.

8. Specific Association of Human Telomerase Activity with Immortal Cells and Cancer. Science. 1994, 226, 2011-2015.

9. Hiyama, E.; Hiyama, K.; Yokoyama, T.; Matsuura, Y.; Piatyszek, M. A.; and Shay, J. W. Correlating Telomerase activity Levels with Human Neuroblastoma Outcomes. Nature Med. 1995, 1, 249-255; DOI 10.1038/nm0395-249. 
10. Hiyama, K.; Hiyama, E.; Ishioka, S.; Yamekido, M.; Inai, K.; Gazdar, A. F.; Piatyszek, M. A.; Shay, J. W. Telomerase Activity in Small-Cell and Non-Small-Cell Lung Cancers. J. Natl. Cancer Inst. 1995, 87, 895-902; DOI 10.1093/jnci/87.12.895; PubMed 7666478.

11. Hiyama, E.; Yokoyama, T.; Tatsumoto, N.; Hiyama, K.; Imamura, Y.; Murakami, Y.; Kodama, T.; Piatyszek, M. A.; Shay, J. W.; Matsuura, Y. Telomerase Activity in Gastric Cancer. Cancer Res. 1995, 55, 3258-3262; PubMed 7614459.

12. Hayflick, L.; Moorhead, P. S. The Serial Cultivation of Human Diploid Cell Strains. Exp. Cell. Res. 1961, 25, 585-621; DOI 10.1016/0014-4827(61)90192-6.

13. Olevnikov, A. M. A Theory of Marginotomy: The Incomplete Copying of Template Margin in Enzymic Synthesis of Polynucleotides and Biological Significance of the Phenomenon. J. Theor. Biol. 1973, 41, 181-190; DOI 10.1016/0022-5193(73)90198-7; PubMed 4754905.

14. Greider, C. W. Telomere Length Regulation. Ann. Rev. Biochem. 1996, 65, 337-365.

15. Saldanha, S. N.; Andrews, L. G.; Tellefsbol, T. O. Analysis of Telomerase Activity and Detection of its Catalytic Subunit, hTERT. Analytical Biochemistry. 2003, 315, 1-21; DOI 10.1016/S00032697(02)00663-2; PubMed 12672407.

16. Potter, J. D. Morphogens, Morphostats, Microarchitecture and Malignancy. Nat. Rev. Cancer. 2007, 7, 464-474; DOI 10.1038/nrc2146; PubMed 17522715.

17. Sack, G. H. Human Cell Transformation by Simian Virus 40. In Vitro. 1981, 17, 1-19; DOI 10.1007/BF02618025; PubMed 6260624.

18. Di Paulo, J. A. Asbestos-Associated Chromosomal Changes in Human. J. Natl. Cancer Inst. 1983, 70, 3-8; PubMed 6571918.

19. Chang, S. E. In Vitro Transformation of Human Epithelial Cells. Biochim. Biophys. Acta. 1986, 823, 161-194; PubMed 2423124.

20. Greider, C. W.; Blackburn, E. H. Identification of a Specific Telomere Terminal Transferase Activity in Tetrahymena Extracts. Cell. 1985, 43, 405-413; DOI 10.1016/0092-8674(85)90170-9; PubMed 3907856.

21. Greider, C. W.; Blackburn, E. H. A Telomeric Sequence in the RNA of Tetrahymena Telomerase Required for Telomere Repeat Synthesis. Nature. 1989, 337, 331-337; DOI 10.1038/337331a0; PubMed 2463488.

22. Zahler, A. M.; Prescott, D. M. Telomere Terminal Transferase Activity in the Hypotrichous Ciliate Oxytricha Nova and a Model for Replication of the Ends of Linear DNA molecules. Nucleic Acids Res. 1988, 16, 6953-6872; DOI 10.1093/nar/16.14.6953; PubMed 3136437.

23. Shippen-Lentz, D.; Blackburn, E. H. Telomere Terminal Transferase Activity for Euplotes Crassus Adds Large Numbers of TTTTGGGG Repeats only Telomeric Primers. Mol. Cell. Biol. 1989, 9, 2761-2764; PubMed 2474761.

24. Autexier, C.; Greider, C. W. Telomerase and Cancer: Revisiting the Telomere Hypothesis. Trends Biochem. 1998, 21, 387-391; DOI 10.1016/0968-0004(96)10042-6.

25. Counter, C. M.; Hirte, H. W.; Bacchetti, S.; Harley, C. B. Telomerase Activity in Human Ovarian Carcinoma. Proc. Natl. Acad. Sci. U. S. A. 1994, 91, 2900-2904; DOI 10.1073/pnas.91.8.2900; PubMed 8159676. 
26. Counter, C. M.; Botelho, F. M.; Wang, P.; Harley, C. B.; Bacchetti, S. Stabilization of Short Telomers and Telomerase Activity Accompany Immortalization of Epstein-Barr VirusTransformed Human B Lymphocytes. J. Virol. 1994, 68, 3410-3414; PubMed 8151802.

27. Fajkus, J. Detection of Telomerase Activity by the TRAP Assay and its Variants and Alternatives. Clinica Chimica Acta. 2006, 371, 25-31; DOI 10.1016/j.cca.2006.02.039.

28. Fajkus, J.; Koppova, K.; Kunicka, Z. Dual-Color Real-Time Telomeric Repeat Amplification Protocol. Biotechniques. 2003, 23, 912-914.

29. Aldous, W. K.; Grabill, N. R. A Fluorescent Method for Detection of Telomerase Activity. Diagn. Mol. Pathol. 1997, 6, 102-110; DOI 10.1097/00019606-199704000-00006; PubMed 9098649.

30. Savoysky, E.; Akamatsu, K.; Tsuchiya, M.; Yamazaki, T. Detection of Telomerase Activity by Combination of TRAP Method and Scintillation Proximity Assay (SPA). Nucleic Acids Res. 1996, 24, 1175-1176; DOI 10.1093/nar/24.6.1175; PubMed 8604356.

31. Ohyashiki, K.; Ohyashiki, J. H.; Nishimaki, J.; et al., Cytological Detection of Telomerase Activity Using an in situ Telomeric Repeat Amplification Protocol Assay. Cancer Res. 1997, 57, 2100 2103; PubMed 9187102.

32. Elmore, L. W.; Forsythe, H. L.; Ferreira-Gonzalez, A.; Garrett, C. T.; Clark, G. M.; Holt, S. E. Real-Time Quantitative Analysis of Telomerase Activity in Breast Tumor Specimens Using a Highly Specific and Sensitive Fluorescent-Based Assay. Diagn. Mo.l Pathol. 2002, 11, 177-185; DOI 10.1097/00019606-200209000-00009.

33. Mayfield, M.P.; Shah, T.; Flannigan, G.M.; Hamilton Stewart ,P. A., ; Bibby, M. C. Telomerase Activity in Malignant and Benign Bladder Conditions. Int. J. Mol. Med. 1998, 1, 835-840; PubMed 9852304.

34. Hoos, A.; Hepp, H. H.; Kaul, S.; Ahlert, T. Telomerase Activity Correlates With Tumor Aggressiveness and Teflects Therapy Effect in Breast Cancer. Int. J. Cancer . 1998, 79, 8-12; DOI 10.1002/(SICI)1097-0215(19980220)79:1<8::AID-IJC2>3.0.CO;2-5; PubMed 9495350.

35. Thurnher, D.; Knerer, B. Non-Radioactive Semiquantitative Testing for the Expression Levels of Telomerase Activity in Head and Neck Squamous cell Carcinomas may be Indicative for Biological Tumour Behaviour. Acta Otolaryngol. 1998, 118, 423-427; DOI 10.1080/00016489850183557; PubMed 9655221.

36. Hirose, M.; Abe-Hashimoto, J.; Ogura, K.; Tahara, H. A Rapid, Useful and Quantitative Method to Measure Telomerase Activity by Hybridization Protection Assay Connected with a Telomeric Repeat Amplification Protocol. J. Cancer Res. Clin. Oncol. 1997, 123, 337-344; PubMed 9222300.

37. Takata, M.; Kerman, K.; Nagatani, N.; Konaka, H.; Namiki, M.; Tamiya, E. Label-Free Bioelectronic Immunoassay for the Detection of Human Telomerase Reverse Transcriptase in Urine. Journal of Electroanalytical Chemistry. 2006, 596, 109-116; DOI 10.1016/j.jelechem.2006.07.025.

38. Nilsson, P.; Persson, B.; Uhlen, M.; Nygren, P.A. Real-Time Monitoring of DNA Manipulations Using Biosensor Technology. Analytical Biochemistry. 1995, 224, 400-408; PubMed 7710099.

39. Schmidt, P. M.; Matthes, E.; Scheller, F. W. Real-Time Determination of Telomerase Activity in Cell Extracts Using an Optical Biosensor. Biol. Chem. 2002, 383, 1659-1666; DOI 10.1515/BC.2002.186; PubMed 12452442. 
40. Matthes, E.; Lehmann, C. Telomerase Protein Rather than its RNA in the Target of Phosphorothioate-Modified Oligonucleotides. Nucleic Acids Res. 1999, 27, 1152-1158; DOI 10.1093/nar/27.4.1152; PubMed 9927750.

41. Schmidt, P. M.; Lehmann, C.; Matthes, E.; Bier, F. F. Detection of Activity of Telomerase in Tumor Cells Using Fiber Optical Biosensors. Biosensors \& Bioelectronics. 2002, 17, 1081-1087; DOI 10.1016/S0956-5663(02)00102-1.

42. Maesawa, C.; Inaba, T.; Sato, H.; Injima, S.; Ishida, K.; Terashima, M.; Sato, R.; Suzuki, M.; Yashima, A.; Ogasawara, S.; Oikawa, H.; Soto, N.; Saito, K.; Masuda, T. A Rapid Biosensor Chip Assay for Measuring the Telomerase Activity Using Surface Plasmon Resonance. Nucleic Acids Research. 2003, 31, 1-6; DOI 10.1093/nar/gng004; PubMed 12519937.

43. Xiao, Y.; Pavlov, V.; Niazov, T.; Dishon, A.; Kotler, M.; Willher, I. Catalytic Beacons for the Detection of DNA and Telomerase Activity. J. Am. Chem. Soc. 2004, 126, 7430-7431; PubMed 15198576.

44. Pavlov, V.; Xiao, Y.; Ron, G.; Dishon, A.; Kotler, M.; Willner, I. Amplified Chemiluminescence Surface Detection of DNA and Telomerase Activity Using Catalytic Nucleic Acid Labels. Anal. Chem. 2004, 76, 2152-2156; DOI 10.1021/ac0352191; PubMed 15053684.

45. Soto, S.; Kondo, H.; Nojima, T.; Takenaka, S. Electrochemical Telomerase Assay with Ferrocenylnaphthalene Diimide as a Tetraplex DNA-Specific Binder. Anal Chem. 2005, 77, 73047309; DOI 10.1021/ac0510235; PubMed 16285679.

46. Pavlov, V.; Willner, I.; Dishon, A.; Kotler, M. Amplified Detection of Telomerase Activity Using Electrochemical and Quartz Crystal Microbalance Measurements. Biosensors \& Bioelectronics. 2004, 20, 1011-1021; DOI 10.1016/j.bios.2004.06.020.

47. Paolsky, F.; Gill, R.; Weizmann, Y.; Mokari, T.; Banin, U.; Willner, I. Lighting-Up the Dynamics of Telomerization and DNA Replication by CdSe-ZnS Quantum Dots. J. Am. Chem. Soc. 2003, 125, 13918-13919; DOI 10.1021/ja035848c; PubMed 14611202.

48. Li, Y. Cds Nanocrystal Induced Chemiluminescence: Reaction Mechanism and Applications. Nanotechnology. 2007, 18, 1-8.

49. Weizmann, Y.; Patolsky, F.; Katz, E.; Willner, I. Amplified Telomerase Analysis by Using Rotating Magnetic Particles: The Rapid and Sensitive Detection of Cancer Cells. ChemBioChem. 2004, 5, 943-948; DOI 10.1002/cbic.200300820; PubMed 15239051.

50. Grimm, J.; Perez, J. M.; Josephson, L.; Weissleder, R. Novel Nanosensors for Rapid Analysis of Telomerase Activity. Cancer Research. 2004, 64, 639-643; DOI 10.1158/0008-5472.CAN-032798; PubMed 14744779.

51. Zheng, G.; Patolsky, F.; Cui, Y. Multiplexed Electrical Detection of Cancer Markers with Nanowire Sensor Arrays. Nature Biotechnology. 2005, 23, 1294-1301; DOI 10.1038/nbt1138; PubMed 16170313.

(C) 2008 by MDPI (http://www.mdpi.org). Reproduction is permitted for noncommercial purposes. 